\title{
PRINCÍPIOS DE UM MODELO BASEADO EM VALORES DE TROCA PARA A AVALIAÇÃO DE SITUAÇÕES DE COOPERAÇÃO *
}

\author{
Antônio Carlos da Rocha Costa ${ }^{1}$
}

\begin{abstract}
Resumo
Este artigo define formalmente o conceito de situação de cooperação e introduz princípios de um modelo baseado em valores de troca para a avaliação desse tipo de situação. Faz-se uma revisão breve das teorias utilizadas (Dependência Social, Delegação/Adoção de Tarefas, Valores de Troca) e tenta-se aproximar as linguagens em que elas são formuladas. Ao final, apresenta-se um conjunto mínimo de requisitos de um sistema de apoio à avaliação de situações de cooperação para ser integrado a ambientes virtuais cooperativos.
\end{abstract}

\section{PRINCIPLES OF AN EXCHANGE VALUE-BASED MODEL FOR THE EVALUATION OF COOPERATION SITUATIONS}

\begin{abstract}
This paper formally defines the concept of cooperation situation and introduces the principles of an exchange value-based model for the evaluation of such type of situations. A brief summary is given of the theories used (Social Dependence, Delegation/Adoption of Tasks, Exchange Values). An attempt is made to approximate the languages in which such theories are formulated. Finally, a minimal set of requirements is presented for systems aiming to support the evaluation of cooperation situations, which systems are to be integrated in virtual cooperative environments.
\end{abstract}

\section{Introdução}

Este artigo visa elaborar um modelo capaz de estruturar de modo operacional a noção de cooperação, no contexto dos sistemas de apoio a atividades em grupo. Ele toma como base três teorias específicas: a Teoria da Dependência Social, a Teoria dos Mecanismos de Delegação e Adoção de Tarefas, e a Teoria dos Valores de Troca. As duas primeiras foram esquematizadas por Cristiano Castelfranchi (1997, 1998). A terceira foi esquematizada por Jean Piaget (1973).

O artigo apresenta uma articulação entre essas três teorias de modo a possibilitar a modelagem sistemática da construção do que chamamos de situações de cooperação,

\footnotetext{
* Trabalho parcialmente financiado pelo CNPq e FAPERGS.

1 Doutor em Ciência da Computação, UFRGS, 1993. Professor adjunto da Escola de Informática e Programa de Pós-graduação em Informática - UCPel. Professor colaborador convidado do Programa de Pós-graduação em Informática na Educação e do Programa de Pós-graduação em Computação UFRGS. Email: rocha@atlas.ucpel.tche.br V.3 $\mathrm{N}^{\circ}$ 2, Novembro, 2005
} 
isto é, situações de dependência em que a cooperação se estabeleceu para possibilitar que os indivíduos nela envolvidos possam realizar seus objetivos.

O fundamento que possibilita a articulação dessas teorias é um modelo cognitivo básico que é compatível com todas elas. Trata-se de uma extensão simples do modelo tradicional de agente deliberativo desenvolvido na área da IA Distribuída.

Esse modelo básico é plenamente compatível com as teorias de Castelfranchi, que utiliza explicitamente o modelo tradicional em todos os seus trabalhos. É também plenamente compatível com o nível de abstração axiomatizante com que Piaget desenvolveu sua Teoria dos Valores de Troca, encaixando-se perfeitamente na classe de modelos formais que esse autor costumava chamar de "modelos cibernéticos".

A Sec. 2 do artigo introduz o modelo cognitivo básico. A Sec. 3 resume a Teoria da Dependência Social e a Teoria da Delegação e Adoção de Tarefas, introduzindo o conceito de situação de cooperação.

A Sec. 4 resume a Teoria das Trocas Sociais e dos Valores de Troca e introduz nosso modelo para a reconstrução lógica de situações de cooperação, permitindo que os valores de troca sejam utilizados tanto nos processos de estabelecimento de situações de cooperação.

A Sec. 5 avança, de modo preliminar, na direção de uma aplicação do modelo de situações de cooperação aos ambientes virtuais de trabalho cooperativo, indicando um conjunto mínimo de requisitos a que tais sistemas devem atender, para poderem dar suporte adequado ao conceito de cooperação, tal como definido aqui. A Sec. 6 traz a Conclusão.

\section{Modelo Cognitivo Básico}

O modelo cognitivo básico de que partimos, e que claramente é compatível tanto com a Sociologia Cognitivista de Cristitano Castelfranchi quanto com a Sociologia Piagetiana, é definido informalmente como segue. Todo indivíduo $\mathbf{A}, \mathbf{B}, \mathbf{C}, \ldots$ é pensado como estando dotado de um aparelho cognitivo constituído de:

- um conjunto de informações sobre si mesmo, o ambiente em que ele se encontra e os outros indivíduos que atuam nesse ambiente; esse conjunto de informações é dito ser o conjunto de crenças que o indivíduo tem sobre aqueles elementos;

- um conjunto de objetivos, que são situações (em si mesmo, no ambiente, nos outros indivíduos) que o indivíduo gostaria que se tornassem realidade; essas situações se caracterizam por presença/ausência de certos elementos, presença/ausência de certas propriedades em certos elementos, presença/ausência de certas relações entre certos elementos;

- um conjunto de planos, ou estratégias, que são essencialmente uma indicação de seqüências de ações que possibilitam ao indivíduo, ao final da realização das mesmas, alcançar um certo objetivo; em geral, para cada objetivo, o indivíduo pode ter disponível um certo número de planos 
(incluindo o caso em que não dispõe de plano nenhum para alcançar um certo objetivo);

- um conjunto de ações que o indivíduo pode realizar sobre si mesmo (internamente), sobre o ambiente e sobre os outros indivíduos, de modo a transforma-los; essas ações podem ser incluídas nos planos do indivíduo, para auxiliá-lo a alcançar seus objetivos;

- um conjunto de sensações, ou percepções, que o indivíduo pode realizar sobre si mesmo (externamente), sobre o ambiente e sobre os outros indivíduos, de modo a obter informações sobre as situações em que esses elementos se encontram, em um determinado momento;

- um conjunto de valores que o indivíduo pode utilizar para avaliar ações, percepções, indivíduos e situações, de modo a auxiliar na sua tomada de decisão sobre as ações a realizar em cada instante;

- um procedimento de tomada de decisão que leve em conta os objetivos atuais do indivíduo, seus planos, crenças, possíveis ações e percepções, bem como o conjunto de avaliações realizadas, para deliberar sobre a seqüência de ações que o agente vai realizar, tendo em vista alcançar aqueles objetivos.

\section{Situações de Dependência e Situações de Cooperação}

\section{Situações de Dependência}

Dado um objetivo a alcançar e um plano para alcança-lo, cada indivíduo deve verificar se todas as tarefas (ações ou sub-seqüências de ações) envolvidas no plano podem ser realizadas por ele, ou se algumas delas devem ser atribuídas a outros indivíduos, para que esses o ajudem na busca daquele objetivo.

Se todas as tarefas envolvidas no plano podem ser realizadas pelo próprio indivíduo, diz-se que esse indivíduo é autônomo para tarefas, relativamente aos demais indivíduos que se encontram no ambiente, juntamente com ele.

Se acontece de algumas das tarefas envolvidas no plano só poderem ser realizadas por outros indivíduos, diz-se que aquele indivíduo é fortemente dependente para tarefas, relativamente aos demais indivíduos. Se acontece de aquele indivíduo ser capaz de realizar todas tarefas mas, por conveniência (fundamentada em razões quaisquer) considerar ser melhor que outros indivíduos realizem algumas delas, diz-se que ele é fracamente dependente para tarefas, relativamente a esses outros indivíduos.

A Fig. 1 mostra uma representação gráfica para algumas situações de dependência. $\mathrm{Na}$ Fig. 1(a), o indivíduo $\mathbf{A}$ tem uma dependência unilateral do indivíduo $\mathbf{B} \mathrm{em}$ relação à tarefa $\mathbf{T}$ incluída em seu plano $\mathbf{P}$. Na Fig. 1(b), os indivíduos $\mathbf{A}$ e $\mathbf{B}$ tem uma dependência bilateral entre si, através de tarefas incluídas em dois de seus planos. A Fig. 1(c) mostra uma situação de dependência mais geral, envolvendo diversos indivíduos. 
a)<smiles>[10BH][In][125I]</smiles>

b)

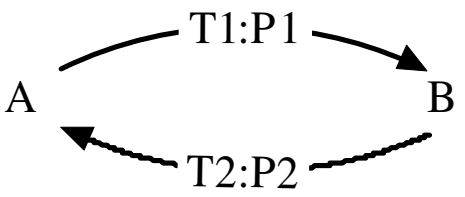

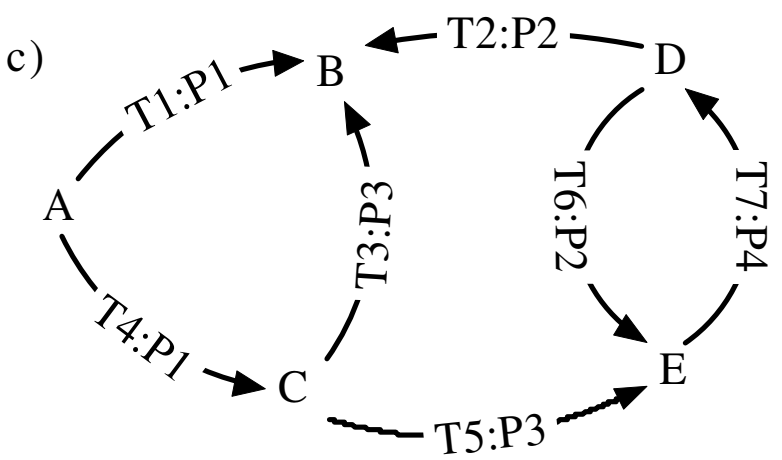

Fig. 1: Situações de Dependência.

\section{Delegação e Adoção de Tarefas}

Chama-se delegação de tarefa o ato pelo qual um indivíduo decide que determinada tarefa envolvida em um dos seus planos será realizada por um outro indivíduo, que não ele próprio. Chama-se adoção de tarefa o ato pelo qual um indivíduo decide aceitar a realização de uma determinada tarefa que lhe foi delegada.

Nota-se que a delegação de tarefas é um ato que, por si só, não tem consequiência sobre o conjunto dos indivíduos, porque é um ato interno ao indivíduo A que delega a tarefa, sem afetar diretamente o indivíduo $\mathbf{B}$ ao qual a tarefa é delgada.

A delegação de tarefas pode ser de dois tipos: a delegação de tarefas é inevitável quando o indivíduo A não tem no seu repertório de ações a totalidade do conjunto de ações necessárias para a realização da tarefa. A delegação de tarefas se dá por conveniência, quando o indivíduo $\mathbf{A}$, apesar de ter em seu repertório de ações todas as ações necessárias à realização da tarefa, avalia que lhe é mais conveniente que a tarefa seja realizada pelo indivíduo $\mathbf{B}$.

Por outro lado, a adoção de tarefas é um ato interno ao indivíduo B ao qual a tarefa foi delegada. Consiste essencialmente em duas etapas: 1) deliberação sobre se ele (B) efetivamente vai realizar a tarefa que lhe foi delegada; e 2) realização efetiva da tarefa, no momento apropriado. Adicionalmente, é possível que B adote uma tarefa de $\mathbf{A}$ sem que esta lhe tenha sido delegada. Chama-se adoção espontânea, esse tipo de adoção de tarefas.

A deliberação sobre se a tarefa delegada vai ser efetivamente realizada, ou não, envolve uma avaliação da situação de cooperação que irá se formar, depois da adoção. Este artigo contribui para o esclarecimento de como os valores de troca envolvidos nas situações de cooperação podem ser utilizados para subsidiar os dois processos de deliberação, tanto o relativo à delegação de tarefas quanto o relativo à adoção de tarefas. 


\section{Situações de Cooperação}

Reconhecida uma situação de dependência, a primeira providência que o indivíduo A deve tomar é a de determinar o conjunto de agentes dos quais é dependente relativamente à diversas tarefas para as quais não tem capacidade (ou interesse) de realizar por si próprio.

A seguir, A deve fazer com que cada um desses indivíduos $\mathbf{B}$ passe a ter a informação de que ele lhe está delegando determinadas tarefas. Essa informação pode ser passada a $\mathbf{B}$ através de diversas formas (solicitação, imposição, etc.).

Uma vez $\mathbf{B}$ adotando a tarefa que lhe foi delegada, estabelece-se entre $\mathbf{A}$ e $\mathbf{B}$ o que se pode chamar de situação de pré-cooperação: o indivíduo A encontra-se em uma situação em que é capaz de se beneficiar de uma tarefa delegada à execução pelo indivíduo B. Uma situação de pré-cooperação é, portanto, uma situação de dependência unilateral em que a dependência de $\mathbf{A}$ em relação a $\mathbf{B}$ foi suprida por um movimento de delegação/adoção da tarefa que determinou a dependência.

Chamamos de situação de cooperação à situação de dependência bilateral entre indivíduos A e $\mathbf{B}$ que foi suprida por um movimento de dupla delegação/adoção, envolvendo tanto a tarefa que criou a dependência de $\mathbf{A}$ em relação a $\mathbf{B}$ quanto a tarefa que criou a dependência de $\mathbf{B}$ em relação a $\mathbf{A}$.

O conceito de situação de cooperação permite formalizar uma distinção conceitual, bastante difundida, entre as idéias gerais de cooperação e de colaboração. Pode-se definir a cooperação entre A e B como a situação resultante de uma dependência bilateral em que os dois indivíduos têm objetivos diferentes, isto é, em que os objetivos das duas tarefas envolvidas são diferentes: objetivo(T) $\neq$ objetivo( $\left(\mathbf{T}^{\prime}\right)$.

Por outro lado, pode-se definir colaboração entre A e B como a situação resultante de uma dependência bilateral em que os dois indivíduos têm o mesmo objetivo, isto é, em que os objetivos das duas tarefas envolvidas são iguais: objetivo(T) = objetivo( $\left.\mathbf{T}^{\prime}\right)$.

Neste trabalho não se adota essa distinção entre cooperação e colaboração, preferindo-se englobar as duas possibilidades no único termo situação de cooperação, por se adotar a definição mais simples, dada por Piaget, de que cooperar é co-operar (operar em conjunto).

\section{Valores de Troca e as Deliberações sobre Situações de Cooperação}

\section{Trocas Sociais e Valores de Troca}

Piaget (1973) privilegia a visão estrutural dos grupos sociais, em que as relações sociais são vistas como trocas de serviços entre os indivíduos participantes do grupo. Entendendo um serviço como uma tarefa realizada por um indivíduo em benefício de outro, é possível identificar plenamente os conceitos de serviço em Piaget e de tarefa em Castelfranchi. 
Assim, é possível aproximar a Teoria da Dependência Social da Teoria dos Valores de Troca, que Piaget elaborou para sustentar uma explicação operatória das trocas sociais e, com ela, sustentar uma explicação operatória das normas sociais, tanto as morais e quanto as jurídicas.

Portanto, é possível revisitar o modelo básico de troca social elaborado por Piaget e reapresenta-lo em termos um pouco mais castelfranchianos. Desse modo, podemos dizer que uma troca social envolve dois indivíduos, A e $\mathbf{B}$, cujas interações podem ser organizadas conforme dois tipos de momentos, chamados etapas I e II.

Nas etapas do tipo I, o indivíduo $\mathbf{A}$ adota (espontaneamente ou não) e realiza uma tarefa do indivíduo B. Nas etapas do tipo II, B adota e realiza uma tarefa de $\mathbf{A}$ (por solicitação de A).

As etapas do tipo I podem ser vistas como a etapas em que um indivíduo $\mathbf{A}$, ao adotar uma etapa delegada por outro indivíduo $\mathbf{B}$, acumula um certo crédito (de tipo moral ou jurídico) que lhe permitirá, posteriormente, cobrar de $\mathbf{B}$ um retorno, isto é, a adoção de uma tarefa qualquer que $\mathbf{A}$ lhe venha a delegar.

A função dos valores de troca nesse processo de troca social é dupla. Por um lado, eles possibilitam que os indivíduos determinem o benefício líquido que estão tendo com essas trocas e, assim, possam deliberar sobre a renovação e a continuidade das suas interações.

Por outro lado, os valores permitem que os indivíduos estabeleçam acordos entre si (regras morais ou jurídicas) possibilitando o acionamento de medidas de reparação cada vez que as trocas se realizam de modo tal que um deles não seja recompensado conforme estabelecido pelo contrato em vigor.

Para tanto, a análise do processo de troca permite definir um certo conjunto de valores de troca e, com eles, estabelecer equações que determinam as condições de equilíbrio das trocas. O conjunto de valores de troca estabelecido por Piaget (Piaget) é o seguinte:

$\mathbf{R}_{\mathbf{I}(\mathbf{A B})}=$ custo da realização da tarefa adotada por $\mathbf{A}$ em favor de $\mathbf{B}$, numa etapa $\mathbf{I}$

$\mathbf{S}_{\mathbf{I}(\mathbf{A B})}=$ benefício (satisfação) obtido por $\mathbf{B}$ com a realização da tarefa por $\mathbf{A}$, em I

$\mathbf{T}_{\mathbf{I}(\mathbf{A B})}=$ dívida adquirida por $\mathbf{B}$, em relação a $\mathbf{A}$, pela tarefa realizada em $\mathbf{I}$

$\mathbf{V}_{\mathbf{I}(\mathbf{A B})}=$ crédito adquirido por $\mathbf{A}$, em relação a $\mathbf{B}$, pela tarefa realizada em $\mathbf{I}$

$\mathbf{V}_{\mathbf{I I}(\mathbf{A B})}=$ crédito cobrado por $\mathbf{A}$ de $\mathbf{B}$, para obtenção de uma adoção de tarefa em II

$\mathbf{T}_{\mathbf{I I}(\mathbf{A B})}=$ dívida reconhecida por $\mathbf{B}$ em relação a $\mathbf{A}$, pela realização da tarefa em $\mathbf{I}$

$\mathbf{R}_{\mathbf{I I}(\mathbf{A B})}=$ custo da tarefa adotada por $\mathbf{B}$ em retorno à tarefa adotada por $\mathbf{A}$ em $\mathbf{I}$

$\mathbf{S}_{\mathbf{I I}(\mathbf{A B})}=$ benefício (satisfação) obtido por $\mathbf{A}$ com a realização da tarefa por $\mathbf{B}$, em II 
A condição de equilíbrio de uma troca é simplesmente a da igualdade de todos esses valores.

Como consequiência dessa igualdade geral, tem-se as duas igualdades particulares mais importantes, $\mathbf{R}_{\mathbf{I}(\mathbf{A B})}=\mathbf{S}_{\mathbf{I I}(\mathbf{A B})}$ e $\mathbf{R}_{\mathbf{I I}(\mathbf{A B})}=\mathbf{S}_{\mathbf{I}(\mathbf{A B})}$, que indicam que os dois indivíduos A e $\mathbf{B}$ obtiveram valores de benefício iguais aos custos despendidos para a realização das tarefas respectivamente adotadas.

Isso significa que, do ponto de vista do balanço dos valores envolvidos nas realizações de seus respectivos planos, os processos de delegação e adoção de tarefas não causaram prejuízos nem ganhos aos indivíduos $\mathrm{A}$ e $\mathrm{B}$, de modo que a avaliação da importância daqueles processos pode se restringir ao seu papel na lógica interna dos referidos planos.

\section{Valores de Troca e as Deliberações sobre Delegação e Adoção de Tarefas}

Do ponto de vista dos valores envolvidos nos processos de delegação e adoção de tarefas, as duas palavras-chave que relevam da análise desses processos feita por Castelfranchi são custo de delegação e custo de adoção.

O custo de delegação de uma tarefa reflete o custo com que o indivíduo $\mathbf{A}$ deve arcar para que a delegação da tarefa ao indivíduo B se efetue. Consiste no somatório dos custos das diversas etapas desse processo, entre eles: 1) custo da comunicação pela qual $\mathbf{A}$ informa $\mathbf{B}$ a tarefa delegada; 2) custo da comunicação pela qual $\mathbf{B}$ informa $\mathbf{A}$ de que a tarefa foi adotada; 3) custo da comunicação pela qual $\mathbf{B}$ informa $\mathbf{A}$ dos resultados da tarefa; 4) no caso de $\mathbf{B}$ recusar preliminarmente a adoção da tarefa, o custo das ações de persuasão ou imposição, que levem $\mathbf{B}$ a adotar a tarefa.

$O$ custo de adoção de uma tarefa reflete o custo com que o indivíduo $\mathbf{B}$ deve arcar para que a tarefa a ele delegada por A se efetue. Consiste no somatório dos custos das diversas etapas desse processo, entre eles: 1) custo da comunicação pela qual B fica informado por A da tarefa a ele delegada; 2) custo da comunicação pela qual $\mathbf{B}$ informa $\mathbf{A}$ de que a tarefa foi adotada; 3 ) custo da comunicação pela qual $\mathbf{B}$ informa $\mathbf{A}$ dos resultados da tarefa; 4) custo da execução da tarefa, propriamente dita; 5) perdas que $\mathbf{B}$ terá com a não execução das tarefas que terá de deixar de executar, para poder executar a tarefa delegada por $\mathbf{A}$.

Podemos chamar de $\mathbf{C d}$ o custo da delegação e de $\mathbf{C a}$ o custo da adoção de uma tarefa. Então, podemos estabelecer formalmente, de modo preliminar, o que Castelfranchi denomina de condições de racionalidade para a delegação e a adoção de tarefas, relacionando-as com os tipos de etapas de trocas, I ou II, em que estão envolvidas.

Assim, em relação ao problema da delegação de tarefas, podemos dizer - em primeira aproximação - que é racional para um indivíduo delegar uma tarefa para outro se e somente se o benefício que ele terá com a delegação é maior do que o custo dessa delegação.

Ao enquadrarmos essa definição de racionalidade de delegação de tarefas nos termos do modelo de trocas piagetiano, vemos que o benefício a que se refere à definição constitui-se nas satisfações que os indivíduos podem ter com as trocas. 
Então, no contexto de uma etapa de troca do tipo $\mathbf{I}$, é racional para o indivíduo $\mathbf{B}$ delegar uma tarefa ao indivíduo A se e somente se $\mathbf{S}_{\mathbf{I}(\mathbf{A B})}>\mathbf{C} \mathbf{d}_{\mathbf{I}(\mathbf{A B})}$. Já no contexto de uma etapa de troca do tipo II, é racional para o indivíduo A delegar uma tarefa ao indivíduo $B$ se e somente se $\mathbf{S}_{\mathbf{I I}(\mathrm{AB})}>\mathbf{C} \mathbf{d}_{\mathbf{I}(\mathbf{A B})}$.

Igualmente, em relação ao problema da adoção de tarefas, podemos dizer - em primeira aproximação - que é racional para um indivíduo adotar uma tarefa para outro se e somente se o benefício que ele terá com a adoção é maior do que o custo dessa adoção.

Ao enquadrarmos essa definição de racionalidade de adoção de tarefas nos termos do modelo de trocas piagetiano, vemos que o benefício a que se refere a definição constitui-se nos créditos que os indivíduos podem adquirir com as trocas e aos débitos que podem pagar com elas.

Assim, no contexto de uma etapa de troca do tipo $\mathbf{I}$, é racional para o indivíduo $\mathbf{A}$ adotar uma tarefa do indivíduo $\mathbf{B}$ se e somente se $\mathbf{V}_{\mathbf{I}(\mathbf{A B})}>\mathbf{C} \mathbf{a}_{\mathbf{I}(\mathbf{A B})}$. Já no contexto de uma etapa de troca do tipo II, é racional para o indivíduo $\mathbf{B}$ adotar uma tarefa do indivíduo A se e somente se $\mathbf{T}_{\mathbf{I}(\mathbf{A B})}>\mathbf{C} \mathbf{d}_{\mathbf{I}(\mathbf{A B})}$.

Uma observação é necessária: é preciso notar que como os valores de troca têm sinais algébricos (podem ser positivos, nulos ou negativos), as comparações indicadas acima devem ser feitas levando em conta apenas as quantidades absolutas dos valores envolvidos (quantidades sem sinal), não seu valor algébrico (com sinal).

\section{A Avaliação das Situações de Cooperação e o Problema das Personalidades}

Os critérios de racionalidade dados por Castelfranchi, indicados na Séc. 4.2, podem ser chamados de critérios de racionalidade sincrônica para o problema da delegação e da adoção de tarefas, porque só levam em consideração os aspectos momentaneamente envolvidos na troca.

Para indivíduos capazes de elaborar uma estratégia para o gerenciamento de seus valores de troca, a racionalidade necessária perpassa trocas envolvendo seqüências constituídas de várias etapas de troca de tipos I e II.

Nesse caso, os critérios de racionalidade devem considerar a evolução temporal dos balanços de valores de troca da cada um deles, constituindo o que se pode chamar de critérios de racionalidade diacrônica para o problema da delegação e da adoção de tarefas.

Tais critérios diacrônicos vão, certamente, muito além dos elementos envolvidos nos critérios de racionalidade sincrônica. Eles exigem que o indivíduo tenha uma estratégia relativamente não apenas à questão da organização lógica do conjunto de ações que deseja executar em seu plano, mas também que tenha uma estratégia de valores de troca, determinando como ele quer que esses valores evoluam ao longo do tempo.

As estratégias diacrônicas estão, então, intimamente ligadas ao problema das personalidades dos agentes. Como Piaget indicou através de diversos exemplos, em (Piaget, 1973), e como Castelfranchi elaborou em diversos trabalhos (ver, p.ex., 
(Castelfranchi, 1977)), as personalidades dos indivíduos condicionam o modo como eles lidam com as trocas que fazem com os outros e, conseqüentemente, com os valores envolvidos nessas trocas.

Definindo personalidade com Castelfranchi (1997), como um conjunto de traços que induzem as atitudes dos indivíduos frente a diversos elementos envolvidos em seus comportamentos, é possível determinar traços de personalidade que induzem diferentes atitudes dos indivíduos frente aos valores de troca.

Assim, podemos determinar diversas atitudes dos indivíduos frente aos valores de troca (egoísta, altruísta, etc.) assim como em relação à questão do equilíbrio dos valores de troca (tolerante, intolerante, etc.). Em (Dimuro et. al., 2005) apresenta-se uma primeira elaboração dessa idéia de análise de personalidades com base nas atitudes em relação aos valores de troca.

\section{Requisitos de um Sistema de Suporte à Avaliação da Cooperação}

Visando dar um primeiro encaminhamento às possibilidades de aplicação da fusão das idéias de Piaget e Castelfranchi, obtida no modelo de valores de troca para situações de cooperação, vamos dar aqui uma indicação dos requisitos do que denominamos sistema de suporte à avaliação da cooperação.

Um sistema de suporte à avaliação da cooperação é um sistema que se pode agregar aos ambientes virtuais de apoio ao trabalho cooperativo, para dotar seus usuários de recursos de informação para uma avaliação crítica do processo de cooperação suportado pelo ambiente.

Tomamos como base trabalhos anteriores (Palazzo et al., 2003), (Silva, 2004) e, principalmente (Costa, 2004), estendemo-los com requisitos determinados pelos conceitos introduzidos neste artigo. Assim, consideramos que um sistema de suporte à avaliação da cooperação deve estar dotado de pelo menos os seguintes recursos e mecanismos:

- um sistema de registro das tarefas envolvidas no ambiente, juntamente com o possível registro das dependências entre os indivíduos, relativamente a essas tarefas, e das delegações e adoções realizadas, para permitir associação adequada de valores de troca a essas delegações e adoções;

- um sistema de registro de balanços de valores de troca, com capacidade de registrar pelo menos o conjunto de valores indicados acima, para cada par de indivíduos que interagem no ambiente;

- um sistema de registro da história desses balanços de troca, para permitir que os indivíduos que interagem no ambiente tenham acesso à evolução temporal desses balanços;

- no caso de os indivíduos que interagem no ambiente serem usuários do sistema, um sistema de visualização que possibilite aos mesmos um acesso qualitativo imediato à evolução dos balanços de valores de troca; 
- um sistema de entrada de valores, pelos quais os indivíduos que interagem no ambiente possam registrar as suas avaliações das etapas de troca que realizaram;

- um sistema de monitoração das ações dos indivíduos no ambiente, registrando as operações que realizam sobre os objetos componentes do mesmo, para que seja possível estabelecer conjuntos de dados de natureza estatística quantitativa, que possam ser relacionados com as avaliações subjetivas qualitativas que constituem os valores de troca.

Recursos adicionais podem ser pensados, como mecanismos de indução de descrições de traços de personalidades, baseados na observação das seqüências de comportamentos dos indivíduos do ambiente, para formação de estimativas de referência que possam ser comparadas com estimativas feitas particularmente por cada indivíduo.

\section{Conclusão}

Este artigo pretendeu iniciar um trabalho de fusão das linguagens utilizadas por Piaget e por Castelfranchi nas suas análises dos processos de troca sociais. As teorias da Dependência Social, da Delegação e Adoção de Tarefas, e dos Valores de Troca, foram brevemente resumidas.

O conceito de situação de cooperação foi introduzido, como o resultado do suprimento de uma situação de dependência com as delegações e adoções necessárias para a realização dos planos dos indivíduos envolvidos nessas situações.

Os critérios de racionalidade de delegação e adoção de tarefas, estabelecido por Castelfranchi, foi mostrado ser um critério de racionalidade sincrônico. Foi introduzido um critério de racionalidade diacrônico para aqueles dois problemas, que leva em consideração a evolução temporal dos balanços de valores de troca. Foi indicado também o papel que as personalidades dos indivíduos desempenham na questão da avaliação das trocas sociais.

Finalmente, foram propostos requisitos mínimos de um mecanismo de apoio à avaliação de situações de cooperação, a ser incorporado a ambientes virtuais de trabalho cooperativo, para instrumentalizar os indivíduos participantes do ambiente nas suas atividades de avaliação das cooperações que eles estabelecem entre si.

$\mathrm{O}$ artigo abre as possibilidades de uma série de trabalhos que podem ser iniciados imediatamente, tanto em nível teórico-conceitual quanto em nível de aplicação. A implementação do mecanismo de apoio à avaliação de situações de cooperação é um deles. Outro é a formalização da idéia de estratégia de valores de troca, levando em conta a fusão de linguagens aqui estabelecida. 


\section{Referências}

CASTELFRANCHI, C.; FALCONE, R. Towards a Theory of Delegation for Agentbased Systems. Robotics and Autonomous Systems, n. 24, pp.141-157, 1998.

CASTELFRANCHI, C.; ROSIS, R.; FALCONE, R.; PIZZUTILO, S. A Testbed for Investigating Personality-based Multiagent Cooperation. Proc. Symp. Logical Approaches to Agent Modeling and Design. pp. 18-22, France, 1997.

COSTA, A. C. R. C. A Inteligência Social Baseada em Valores de Troca e os Ambientes Cooperativos. In: VII Oficina de Inteligência Artificial. Pelotas, ESIN/UCPel, 23-24/Outubro/2004.

DIMURO, G. P.; COSTA, A. C. R.; GONÇALVES, L. V. Analyzing Personality-Based Multiagent Social Exchanges via Hidden Markov Models. (Submetido para publicação). Disponível em: <http://gmc.ucpel.tche.br/valores>. Acesso em: 23 out. 2005.

PAlAZZO, R. V.; COSTA, A. C. R.; DIMURO, G. P. Proposta de um Sistema de Valores de Troca para o ambiente ENSINET. In: VI Oficina de IA, UCPel, Outubro/2003.

SILVA, E. M. ENSINET/MAI - Ferramenta de Monitoração e Análise das Interações para o ENSINET. Pelotas, dezembro/2004. (Trabalho de Conclusão de Curso). 84p.

PIAGET, J. Estudos Sociológicos. Rio de Janeiro, Forense, 1973. 